\title{
Genitofemoral neuralgia: adding to the burden of chronic vulvar pain
}

This article was published in the following Dove Press journal:

Journal of Pain Research

30 November 2015

Number of times this article has been viewed

\author{
Hans Verstraelen' \\ Eline De Zutter' \\ Martine De Muynck ${ }^{2}$ \\ 'Department of Obstetrics and \\ Gynaecology, Vulvovaginal Disease \\ Clinic, Ghent University Hospital, \\ Ghent, Belgium; ${ }^{2}$ Department of \\ Physical Medicine and Rehabilitation, \\ Ghent University Hospital, Ghent, \\ Belgium
}

\begin{abstract}
The vulva is a particularly common locus of chronic pain with neuropathic characteristics that occurs in women of any age, though most women with neuropathic type chronic vulvar pain will remain undiagnosed even following multiple physician visits. Here, we report on an exemplary case of a middle-aged woman who was referred to the Vulvovaginal Disease Clinic with debilitating vulvar burning and itching over the right labium majus that had been persisting for 2 years and was considered intractable. Careful history taking and clinical examination, followed by electrophysiological assessment through somatosensory evoked potentials was consistent with genitofemoral neuralgia, for which no obvious cause could be identified. Adequate pain relief was obtained with a serotonin-noradrenaline reuptake inhibitor and topical gabapentin cream. We briefly discuss the epidemiology, diagnosis, and treatment of genitofemoral neuralgia and provide a series of clues to guide clinicians in obtaining a presumptive diagnosis of specific neuropathic pain syndromes that may underlie chronic vulvar pain. We further aim to draw attention to the tremendous burden of chronic, unrecognized vulvar pain.
\end{abstract}

Keywords: vulvar pain, genitofemoral nerve, neuropathic pain, vulvodynia, vulvar disease

\section{Introduction}

Neuropathic pain is an umbrella term that has recently been redefined by the International Association for the Study of Pain as pain caused by a lesion or disease of the somatosensory system, ${ }^{1}$ thereby encompassing a number of diverse conditions and body sites. Although largely underappreciated, the vulva is a particularly common locus of chronic pain with neuropathic characteristics in women of any age. In the largest community-based study conducted thus far, chronic "unexplained" vulvar pain, defined as burning or sharp knife-like pain or pain on contact, that lasted for at least 3 months or longer was reported by nearly 7\% of women aged 18-64 years in a US urban area. ${ }^{2}$ In spite of this high prevalence, it was also revealed in the latter study that no proper diagnosis was provided to a majority of women suffering from chronic vulvar pain even following multiple physician visits, ${ }^{2}$ a finding that has been corroborated by several recent US population-based studies. ${ }^{3,4}$ Here, we report on a case of a middle-aged woman with chronic vulvar neuropathic pain that, although unique from a clinical perspective, exemplifies the burden of unrecognized chronic vulvar pain from a public health view, as discussed further.

\section{Case presentation}

A 45-year-old woman was referred to the Vulvovaginal Disease Clinic with debilitating vulvar burning and itching over the right labium majus, also causing severely 
disturbed sleep. Vulvar pain had been persisting for 2 years and was considered intractable. Careful history taking revealed that she had two vaginal deliveries and was known to suffer from Crohn's disease involving the right hemicolon, terminal ileum, and rectum, for which she was treated with azathioprine orally and rectally applied mesalazine. The patient also had a laparoscopic fallopian tube ligation. Finally, she had a history of atopy, and reported occasional seasonal allergic rhinitis symptoms.

The patient was initially followed at a regional hospital. A blind biopsy was taken from the right labial skin in a dermatology practice; it showed no epidermal or dermal anomalies. Several topical treatments were initiated empirically, including vaginal antibiotic and fungistatic agents and various corticoid ointments, without providing relief. During a surgical consult, a nodule was detected in the right labium majus and was the presumed cause of the chronic pain. It was removed under general anesthesia, and had histological features indicative of a ruptured hair follicle. As no diagnosis was obtained, the patient was also asked to discontinue all medications, including those for treatment of Crohn's disease. The patient was eventually referred to our hospital, which is a tertiary referral center.

Careful examination of the vulva and vagina revealed neither visible anomalies nor any other findings indicative of vulvar inflammatory, infectious, or neoplastic mucocutaneous disease. During the clinical exam, the patient was asked to point out the pain area, showing the right labiocrural fold, the anterior surface of the right labium majus, and right medial upper thigh, though burning sensation of the right labium majus was the most prominent complaint and was initially mentioned by the patient as the only complaint. Tactile sense assessment was performed with a piece of cotton wool, a cotton-tip swab, and a sharp wooden stick over these areas and further compared with the contralateral sites. While varied dysesthetic tactile responses were obtained, the overall pattern was hypoesthesia upon soft touch and pronounced hyperesthesia and allodynia upon sharp touch. Though often difficult to differentiate from ilioinguinal neuralgia, this particular pattern was highly suggestive of genitofemoral neuralgia, involving both the genital and femoral branches of the genitofemoral nerve (Figure 1). Maximal passive hip flexion further provoked burning over the right labium majus, though not the thigh.

Subsequently, electrophysiological examination through somatosensory evoked potentials (SSEP) assessment of the labia majora was performed. The labia majora were stimulated with bipolar surface electrodes (cathode proximally) at an intensity of two times the sensory threshold, with stimulus

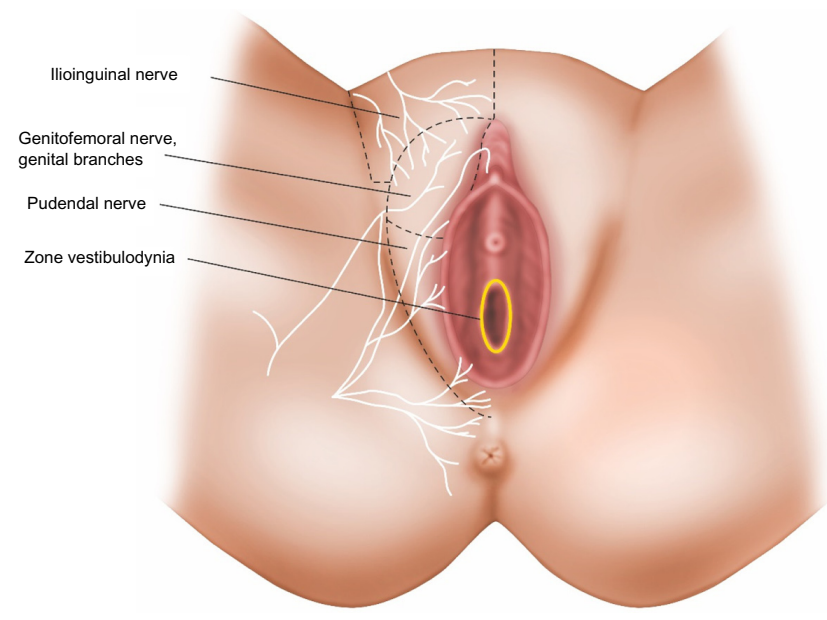

Figure I Topography of neuropathic-type chronic vulvar pain.

Notes: The figure shows the anatomy of the female anogenital region, its primary cutaneous nerves (white) with accompanying innervation zones (dashed lines), and the most common location of vulvodynia, ie, the vulvar vestibule (yellow). Cutaneous nerve distribution in this anatomical region is however highly variable with numerous anatomical variants having been described, complicating clinical diagnosis of neuropathic pain origin in this anatomical region.

duration of $0.2 \mathrm{~ms}$ and a frequency of $2 \mathrm{~Hz}$. Averaging 256 sweeps, responses were recorded twice to ensure reproducibility. Recording was done using needle electrodes, with the active electrode placed $2 \mathrm{~cm}$ behind the $\mathrm{Cz}$ position of the International 10-20 system and the reference electrode at the Fpz position. The ground electrode was placed over the right anterior thigh. Sensitivity was set at $10 \mu \mathrm{V}$ and analysis time at $100 \mathrm{~ms}$. Recordings were performed with the Medelec Sapphire device (Vickers Healthcare Co, Woking, UK). SSEP responses over the right compared to the left labium majus showed a prolonged latency (32.2 versus $29.1 \mathrm{~ms}$ corresponding to $\sim 10 \%$ prolongation) and considerably decreased amplitude $(0.50$ versus $1.58 \mu \mathrm{V}$ corresponding to $\sim 70 \%$ lower amplitude).

Additionally, imaging of the pelvis was conducted through magnetic resonance imaging (MRI), whereby T1-, T2- and proton density-weighted sequences were obtained, though no anomalies could be documented.

Initial pain relief was procured through application of a $5 \%$ lidocaine patch fit to the right labium majus and left in place during daytime for 12 hours a day. While providing some attenuation of pain during the day, the patient continued to experience severely disturbed sleep. Nortriptyline at a daily dose of $25 \mathrm{mg}$ was ill-tolerated without any effect on vulvar pain. Venlafaxine was slowly titrated, starting with a dose of $37.5 \mathrm{mg}$ and adding up in increments of $37.5 \mathrm{mg}$ per day at intervals of 7 days, until a daily dose of $150 \mathrm{mg}$ was reached. While reporting mild side effects of vivid dreaming and increased transpiration, 
the patient experienced rapid improvement of vulvar pain, which allowed her to resume normal sleep at night, after 2 years of sleep deprivation. She gradually tapered off use of the lidocaine patch and reported that she no longer experienced vulvar discomfort during activities of daily life. In view of the side effects under venlafaxine, the treatment regimen was changed to duloxetine $60 \mathrm{mg}$ a day, resulting in disappearance of the side effects, while maintaining optimal pain relief of the vulva. Discomfort over the right thigh, primarily described as itching rather than burning, did persist however, for which we initiated a compounded topical gabapentin cream in a $6 \%$ concentration formulated in a nonionic cream, resulting in significant relief of discomfort over the right thigh. At some point in time during follow-up, the patient reported a slight but hindering exacerbation of vulvar pain under duloxetine. Careful history taking revealed that the pain reoccurred following a bicycle day trip, and the patient was therefore instructed on vulvar care with emphasis on limiting vulvar mechanical strain as a known aggravating factor. One year after initial assessment, the patient remains pain free under continued treatment with duloxetine orally $60 \mathrm{mg}$ a day and $6 \%$ gabapentin cream applied twice daily. For this kind of approach or publication, Ghent University Hospital Institutional Review Board approval was not deemed necessary by our institution. Written informed consent from the patient was obtained.

\section{Discussion}

According to the International Society for the Study of Vulvovaginal Disease, vulvar pain can broadly be classified as pain related to a specific disorder, either as pain occurring in the absence of relevant visible findings or a specific clinically identifiable, neurologic disorder. ${ }^{5}$ Vulvar pain attributable to a specific disorder basically encompasses pain resulting from infectious disease (eg, herpes genitalis), inflammatory mucocutaneous disease (eg, lichen planus), neoplastic disease (eg, squamous cell carcinoma), and neurological disease (eg, postherpetic neuralgia). ${ }^{5}$ In the event that no such underlying disease to vulvar pain can be identified, the term vulvodynia has been adopted, ${ }^{5}$ though etymologically referring to vulvar pain as such. Vulvodynia involves in the vast majority of patients mechanical allodynia and hyperalgesia of the vaginal vestibular area, thought to relate at least in part to marked regional $\mathrm{C}$-fiber hyperinnervation and possibly neuroinflammation. ${ }^{6}$ While the nosology of vulvodynia has been a long-standing matter of debate, ${ }^{5,7,8}$ clearly, the signs and symptoms in vulvodynia show considerable overlap with neuropathic pain, ${ }^{9}$ as assessed by available screening tools. ${ }^{10}$

Women presenting with chronic nociceptive vulvar pain typically elicit visible epithelial alterations (eg, lichen planus, vulvar intraepithelial neoplasia, and genitourinary syndrome of menopause) prompting clinical diagnosis, though histopathological confirmation following biopsy might be sought, and hence most of these, often older patients, will eventually be managed accordingly. Paradoxically, women presenting with the more common neuropathic type of chronic vulvar pain, resulting from vulvodynia or specific neuropathic pain syndromes, usually do not show visible anomalies, and either do not seek medical attention or remain undiagnosed even following multiple physician visits. ${ }^{3,4,11}$ A recent populationbased study revealed, for instance, that merely $3 \%$ of women meeting vulvodynia criteria were actually diagnosed with vulvodynia. $^{3}$

Illustrating this point is the significant diagnostic delay observed in the case we presented, as well as the strong physician tendency to attribute the pain reported by the patient to visible or demonstrable tissue alterations, prompting biopsy and surgery, as well as the number of treatments directed toward infectious and inflammatory conditions, even though no such diagnosis was obtained.

While chronic "unexplained" vulvar pain is a debilitating condition affecting a vast number of women of all ages, ${ }^{2}$ this has been mostly attributed to vulvodynia epidemiology, and hence it has not been established how often chronic vulvar pain is associated with specific neuropathic pain syndromes related to neuralgia of the genitofemoral, ilioinguinal, and pudendal nerves (Figure 1), though not a rarity at a vulvar disease clinic in our experience.

Genitofemoral neuralgia was first described by Magee ${ }^{12}$ as genitofemoral causalgia, and subsequently redefined by Lyon, ${ }^{13}$ where the current nomenclature was introduced. ${ }^{14}$ Genitofemoral neuralgia in women is almost exclusively known as an iatrogenic pain disorder, complicating common lower abdominal and pelvic surgical procedures such as inguinal herniorrhaphy, cesarean section, hysterectomy, and lymph node biopsy, as recently and comprehensively reviewed by Cesmebasi et al. ${ }^{14}$ In the original case series by Magee, ${ }^{12}$ there is also mention of an 11-year-old girl who developed genitofemoral neuralgia after falling while bicycling, presumably involving direct trauma to the groin by the handle bar. ${ }^{13}$ Murovic et al, ${ }^{15}$ in their series of ten patients, also reported on four patients who developed genitofemoral neuralgia after blunt abdominal trauma. We identified only one case report on spontaneous-onset genitofemoral neuralgia, 
not related to surgery or blunt trauma, involving a 20-year-old woman, in which nerve compression was suspected to result from tight-fitting clothing. ${ }^{16}$ Interestingly, also with regard to the case presented by us, bicycle riding has also been reported as a possible factor evoking genitofemoral neuralgia. ${ }^{14}$ Tight-fitting clothing and bicycling are often reported as aggravating or evoking factors in patients with vulvodynia or neuropathic pain attending our vulvar disease clinic, pointing at vulvar mechanical strain through pressure and friction as an important aggravating and possibly initiating factor to chronic vulvar disease, though this is poorly addressed in the medical literature. We cannot explain how the patient presented developed genitofemoral neuralgia, though possibly resulting from a tubal ligation procedure. A relation ship with Crohn's disease could not be established.

The mainstay to the diagnosis of genitofemoral neuralgia has been the use of selective nerve blocks, as originally proposed by Lichtenstein et al. ${ }^{17}$ Historically, this technique involved an essentially blind block near the pubic tubercle, distal to the presumed site of injury following most types of surgery, and with a considerable potential for injury to the surrounding structures at the injection site. Due to the retroperitoneal location dorsal to the psoas muscle, the genitofemoral nerve has been notoriously difficult to target more proximally. Recently, several medical imaging-guided transpsoas techniques have been described by use of computed tomography and ultrasound; ${ }^{18-21}$ magnetic resonance neurography-guided nerve blocks are likely to serve as another excellent diagnostic tool. ${ }^{22}$ In case of clinical suspicion of genitofemoral, ilioinguinal, or pudendal neuralgia, we initially obtain electrophysiological assessment through SSEP, an approach that has not been reported in this context in the literature before. We identified one case report in which ilioinguinal nerve entrapment was suspected through electromyography in a 35-year-old man with acute scrotal pain. ${ }^{23}$ Clearly, the sensitivity of electrophysiological approaches needs to be validated in sufficiently large patient series; however, it has proven a useful noninvasive first-line diagnostic assessment in our experience.

A myriad of therapeutic approaches for genitofemoral neuralgia have been described as comprehensively reviewed by Cesmebasi et al, ${ }^{14}$ including ultrasound-guided nerve block injections, ${ }^{20,21}$ various ablative techniques for neurolysis, ${ }^{24}$ and genitofemoral neurectomy, recently described through a minimally invasive endoscopic retroperitoneal approach. ${ }^{25-27}$ It may be acknowledged that neurolysis or neurectomy of the genitofemoral nerve may be accompanied by hypoesthesia over the labium majus, ${ }^{15}$ possibly impairing sexual function. ${ }^{28}$
Noninvasive, pharmacotherapeutic approaches may provide proper pain relief in a substantial proportion of patients. A recent systematic review by the Special Interest Group on Neuropathic Pain of the International Association for the Study of Pain found sufficient evidence to strongly recommend tricyclic antidepressants, serotonin-noradrenaline reuptake inhibitors, pregabalin, and gabapentin as first-line treatments in neuropathic pain. ${ }^{29}$ In the patient presented here, as with many other patients presenting with chronic vulvar pain, satisfactory pain relief was obtained with serotoninnoradrenaline reuptake inhibitors and topical gabapentin.

In summary, it is widely observed that although many women of all ages suffer from chronic neuropathic type vulvar pain, most of these will remain undiagnosed even following multiple physician visits. A majority of these patients presumably have vulvodynia, while an unknown number of women suffer from a specific neuropathic vulvar pain condition. Although physicians such as dermatologists, urologists, and gynecologists are largely unfamiliar with these conditions, several clues to the diagnosis of genitofemoral, ilioinguinal, or pudendal neuralgia may be particularly useful in the first- or second-line assessment when confronted with the chronic vulvar pain patient (Table 1): 1) careful history taking reveals continuous vulvar pain, often reported as a burning sensation (though possibly also involving itch, tingling, or sharp pain); 2) pain or discomfort is typically unilateral; 3) vulvar pain or discomfort may be most pronounced while sitting, and least prominent when lying; 4) clinical and

Table I Clues to vulvar neuralgia diagnosis in the patient with chronic vulvar pain

\section{History taking}

I. Continuous vulvar pain, possibly disturbing sleep at night

2. Pain character often described as burning, though possibly also involving itch, tingling, or sharp pain

3. Pain interferes with activities of daily life

4. Dyspareunia possibly present, but not obligate

5. Pain often increases upon pressure of friction imposed to the vulvar area (pants, sitting, bicycle riding, etc)

6. Postural gradient in pain intensity may be present: pain most pronounced while sitting, less when standing, and least while lying down Clinical examination

I. Inspection of the vulva does not reveal any relevant anomalies

2. Even if anomalies are observed, these do not necessarily correlate with subjective symptoms

3. Pain is typically unilateral

4. Pain conferred to a well-described vulvar area

5. Pain possibly exacerbated by maximal hip flexion

6. Tactile sense testing over the vulvar area referred often reveals aberrant sensory responses (in comparison to the contralateral site), including allodynia, hyperesthesia, and hypoesthesia 
microbiological analysis does not reveal anomalies, and even if anomalies are observed, these do not necessarily correlate with subjective symptoms, especially since symptoms are unilateral; 5) vulvar pain may be provoked by maximal hip flexion; and 6) tactile sense anomalies may be demonstrated by the use of ordinary disposables available in most physician offices. Finally, first-line treatment may be initiated according to current evidence with tricyclic antidepressants, serotonin-noradrenaline reuptake inhibitors, pregabalin, and gabapentin, whereby it is crucial to inform the patient on the treatment rationale, expected treatment success, and possible side effects.

\section{Acknowledgments}

We would like to thank radiologist Pieter De Visschere for carefully reviewing the MRI images. We are also greatly indebted to Jan De Groote for his valuable contribution in designing the illustration.

\section{Disclosure}

The authors report no conflicts of interest in this work.

\section{References}

1. Jensen TS, Baron R, Haanpää M, et al. A new definition of neuropathic pain. Pain. 2011;152(10):2204-2205.

2. Harlow BL, Stewart EG. A population-based assessment of chronic unexplained vulvar pain: have we underestimated the prevalence of vulvodynia? J Am Med Womens Assoc. 2003;58(2):82-88.

3. Reed BD, Harlow SD, Sen A, et al. Prevalence and demographic characteristics of vulvodynia in a population-based sample. Am J Obstet Gynecol. 2012;206(2):170. e1-e9.

4. Harlow BL, Kunitz CG, Nguyen RH, Rydell SA, Turner RM, MacLehose RF. Prevalence of symptoms consistent with a diagnosis of vulvodynia: population-based estimates from 2 geographic regions. Am J Obstet Gynecol. 2014;210(1):40. e1-e8.

5. Moyal-Barracco M, Lynch PJ. 2003 ISSVD terminology and classification of vulvodynia: a historical perspective. J Reprod Med. 2004;49(10): 772-777.

6. Wesselmann U, Bonham A, Foster D. Vulvodynia: current state of the biological science. Pain. 2014;155(9):1696-1701.

7. Micheletti L, Radici G, Lynch PJ. Provoked vestibulodynia: inflammatory, neuropathic or dysfunctional pain? A neurobiological perspective. J Obstet Gynaecol. 2014;34(4):285-288.

8. Micheletti L, Radici G, Lynch PJ. Is the 2003 ISSVD terminology and classification of vulvodynia up-to-date? A neurobiological perspective. J Obstet Gynaecol. 2015;17:1-5.

9. Eppsteiner E, Boardman L, Stockdale CK. Vulvodynia. Best Pract Res Clin Obstet Gynaecol. 2014;28(7):1000-1012.

Journal of Pain Research

\section{Publish your work in this journal}

The Journal of Pain Research is an international, peer-reviewed, open access, online journal that welcomes laboratory and clinical findings in the fields of pain research and the prevention and management of pain. Original research, reviews, symposium reports, hypothesis formation and commentaries are all considered for publication.
10. Mulvey MR, Bennett MI, Liwowsky I, Freynhagen R. The role of screening tools in diagnosing neuropathic pain. Pain Manag. 2014;4(3): 233-243.

11. Harlow BL, Wise LA, Stewart EG. Prevalence and predictors of chronic lower genital tract discomfort. Am J Obstet Gynecol. 2001;185(3): $545-550$.

12. Magee RK. Genitofemoral causalgia: a new syndrome. Can Med Assoc J. 1942;46(4):326-329.

13. Lyon EK. Genitofemoral causalgia. Can Med Assoc J. 1945;53(3): 213-216.

14. Cesmebasi A, Yadav A, Gielecki J, Tubbs RS, Loukas M. Genitofemoral neuralgia: a review. Clin Anat. 2015;28(1):128-135.

15. Murovic JA, Kim DH, Tiel RL, Kline DG. Surgical management of 10 genitofemoral neuralgias at the Louisiana State University Health Sciences Center. Neurosurgery. 2005;56(2):298-303.

16. O'Brien MD. Genitofemoral neuropathy. Br Med J. 1979;1(6170): 1052.

17. Lichtenstein IL, Shulman AG, Amid PK, Montllor MM. Cause and prevention of postherniorrhaphy neuralgia: a proposed protocol for treatment. Am J Surg. 1988;155(6):786-790.

18. Parris D, Fischbein N, Mackey S, Carroll I. A novel CT-guided transpsoas approach to diagnostic genitofemoral nerve block and ablation. Pain Med. 2010;11(5):785-789.

19. Hackworth RJ, Nagel EJ, Slotto JG. Computed tomographic-guided genitofemoral nerve block: a simple anterior approach. J Comput Assist Tomogr. 2015;39(2):295-297.

20. Peng P, Tumber P. Ultrasound-guided interventional procedures for patients with chronic pelvic pain - a description of techniques and review of literature. Pain Physician. 2008;11:215-224.

21. Shanthanna H. Successful treatment of genitofemoral neuralgia using ultrasound guided injection: a case report and short review of literature. Case Rep Anesthesiol. 2014;2014:371703.

22. Fritz J, Chhabra A, Wang KC, Carrino JA. Magnetic resonance neurography-guided nerve blocks for the diagnosis and treatment of chronic pelvic pain syndrome. Neuroimaging Clin N Am. 2014;24(1): 211-234.

23. ter Meulen BC, Peters EW, Wijsmuller A, Kropman RF, Mosch A, Tavy DL. Acute scrotal pain from idiopathic ilioinguinal neuropathy: diagnosis and treatment with EMG-guided nerve block. Clin Neurol Neurosurg. 2007;109(6):535-537.

24. Campos NA, Chiles JH, Plunkett AR. Ultrasound-guided cryoablation of genitofemoral nerve for chronic inguinal pain. Pain Physician. 2009;12(6):997-1000.

25. Giger U, Wente MN, Büchler MW, Krähenbühl S, Lerut J, Krähenbühl L. Endoscopic retroperitoneal neurectomy for chronic pain after groin surgery. Br J Surg. 2009;96(9):1076-1081.

26. Song JW, Wolf JS Jr, McGillicuddy JE, Bhangoo S, Yang LJ. Laparoscopic triple neurectomy for intractable groin pain: technical report of 3 cases. Neurosurgery. 2011;68(2 Suppl Operative):339-346.

27. Chen DC, Hiatt JR, Amid PK. Operative management of refractory neuropathic inguinodynia by a laparoscopic retroperitoneal approach. JAMA Surg. 2013;148(10):962-967.

28. Martin-Alguacil N, Schober J, Kow LM, Pfaff D. Arousing properties of the vulvar epithelium. $J$ Urol. 2006;176(2):456-462.

29. Finnerup NB, Attal N, Haroutounian S, et al. Pharmacotherapy for neuropathic pain in adults: a systematic review and meta-analysis Lancet Neurol. 2015;14(2):162-173.

\section{Dovepress}

The manuscript management system is completely online and includes a very quick and fair peer-review system, which is all easy to use. Visit http://www.dovepress.com/testimonials.php to read real quotes from published authors. 